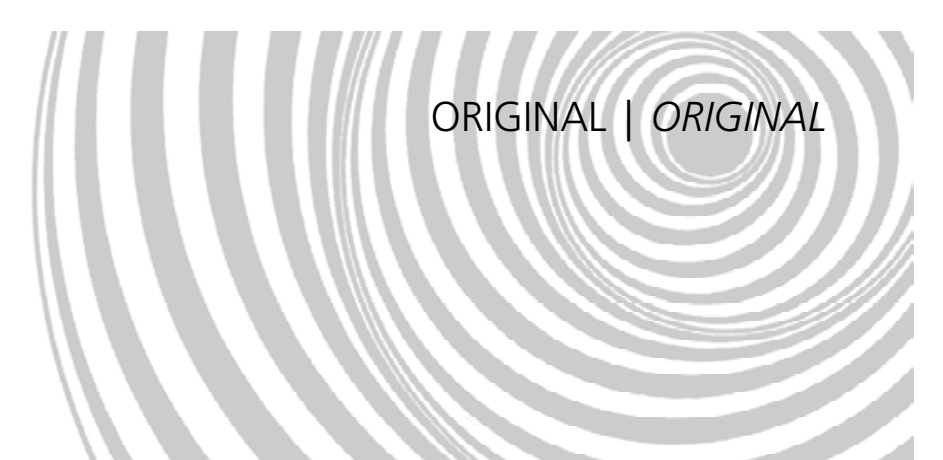

\title{
Validação de um questionário de freqüência de consumo alimentar para população adulta
}

\author{
Validation of a food frequency questionnaire \\ for the adult population
}

\author{
Aída Calvão RIBEIRO ${ }^{1,2}$ \\ Karin Eleonora Oliveira SÁVIO² \\ Maria de Lourdes Carlos Ferreirinha RODRIGUES² \\ Teresa Helena Macedo da COSTA ${ }^{2}$ \\ Bethsáida de Abreu Soares SCHMITZ²
}

\section{RE S U M O}

\section{Objetivo}

Investigar a validade e a reprodutibilidade de um questionário de freqüência de consumo alimentar desenvolvido para a população adulta.

\section{Métodos}

Foram entrevistados, em três momentos, 69 indivíduos, de ambos os sexos, freqüentadores de restaurantes industriais em dois órgãos públicos de Brasília, Distrito Federal. Na primeira e terceira entrevistas foram aplicados um recordatório 24 horas e um questionário de freqüência de consumo alimentar, e na segunda entrevista, um recordatório 24 horas. A duração média do estudo foi de 181 dias. Macronutrientes, vitaminas A e C, cálcio, ferro, zinco, colesterol, fibras e energia total foram analisados utilizando-se os coeficientes de correlação intraclasse e de Pearson, este último na forma bruta e deatenuada.

\section{Resultados}

Quanto à reprodutibilidade, o questionário de freqüência de consumo alimentar obteve desempenho adequado, apresentando resultados acima de 0,7 para lipídeo, colesterol, energia e proteína. Para a validade, os melhores coeficientes deatenuados foram observados para vitamina $C(r=0,66)$, ferro $(r=0,58)$, proteína $(r=0,55)$ e carboidrato $(r=0,55)$ e os piores, para colesterol $(r=0,32)$ e vitamina A $(r=0,37)$.

\section{Conclusão}

A obtenção de coeficientes de correlação expressivos para alguns nutrientes indica que este questionário de freqüência de consumo alimentar constitui-se em um bom instrumento de pesquisa para estudos

\footnotetext{
1 Curso de Nutrição, Universidade de Brasília. Brasília, DF, Brasil.

2 Departamento de Nutrição, Faculdade de Ciências da Saúde, Universidade de Brasília. Campus Universitário Darcy Ribeiro, Asa Norte, 70910-900, Brasília, DF, Brasil. Correspondência para/Correspondence to: A.C. RIBEIRO. E-mail: <calvaoribeiro@hotmail.com>.
} 
epidemiológicos em população adulta, podendo fornecer informações importantes para a implementação de ações na área de saúde e nutrição.

Termos de indexação: consumo alimentar; inquéritos nutricionais; recordatório 24 horas; reprodutibilidade dos testes.

\section{A B S T R A C T}

\section{Objective}

The aim of the present study was to investigate the validity and reproducibility of a food frequency questionnaire developed for the adult population.

\section{Methods}

Sixty-nine individuals from both genders who attended industrial restaurants from two government divisions in Brasilia, Federal District, Brazil, were interviewed on three different occasions. In the first and third interviews, a $24 \mathrm{~h}$ recall and a food frequency questionnaire were applied, and in the second interview only a $24 \mathrm{~h}$ recall was applied. The study lasted 181 days. Macronutrients, vitamins A and C, calcium, iron, zinc, cholesterol, fibers and total energy were analyzed using the intraclass and Pearson's correlation coefficients, this last one in a crude and deattenuated manner.

\section{Results}

Regarding reproducibility, the food frequency questionnaire provided adequate results, presenting correlation coefficients above 0.7 for lipid, cholesterol, energy and protein. For validity, the best deattenuated coefficients were observed for vitamin $C(r=0.66)$, iron $(r=0.58)$, protein $(r=0.55)$ and carbohydrate $(r=0.55)$ and the worst for cholesterol ( $r=0.32)$ and vitamin $A(r=0.37)$.

\section{Conclusion}

The expressive correlation coefficients obtained for some nutrients indicates that this food frequency questionnaire constitutes a good research instrument for epidemiological studies in the adult population, as it can supply important dietary information allowing the implementation of actions in the health and nutrition areas.

Indexing terms: food consumption; nutrition surveys; 24-hour recall; reproducibility of results.

\section{NTRO D U ÇÃ O}

Dois métodos investigativos bastante utilizados em pesquisas populacionais, para a avaliação do consumo, são o questionário de freqüência de consumo alimentar (QFCA) e o recordatório de 24 horas (R24h).

Ambos os métodos apresentam vantagens e desvantagens. O questionário de freqüência é um método relativamente rápido e de baixo custo, que possibilita a classificação conforme níveis de consumo habitual ${ }^{1}$.

Não há um método que possa ser considerado "padrão-ouro" para quantificar o consumo alimentar com grande precisão ${ }^{2,3}$. O recordatório de 24 horas não representa a ingestão habitual, é dependente da memória do entrevistado e necessita de um entrevistador treinado. O questionário de freqüência pode perder detalhes da ingestão, sua quantificação, por vezes, é pouco exata e requer a memória de hábitos do passado'. Aspectos como etnicidade, grau de escolaridade e idade do entrevistado também podem influenciar a fidedignidade das informações colhidas pelo QFCA ${ }^{4}$.

Apesar de todas essas limitações, alguns estudos sobre validação de métodos de inquérito dietético afirmam que o questionário de freqüência alimentar ainda se constitui um dos melhores métodos de investigação nutricional em estudos epidemiológicos, pois suas vantagens tornam possível sua aplicabilidade em pesquisas de grande porte, além de possuir relativa confiabilidade ${ }^{5-8}$. 
O questionário de freqüência alimentar é, freqüentemente, comparado a outros tipos de inquéritos, como recordatórios $24 \mathrm{~h}$ e registros alimentares ${ }^{6,9}$, e com marcadores biológicos ${ }^{3,7,8}$. Existem ainda aqueles que comparam questionários desenvolvidos por outros estudiosos e que se tornaram métodos-padrão, como, por exemplo, "Block" versus "Willett"10,11.

Face à importância e à necessidade de métodos adequados de avaliação do consumo alimentar, o presente trabalho procurou verificar a reprodutibilidade e a validade de um questionário de freqüência alimentar semiquantitativo, elaborado para pesquisas de análise nutricional e consumo alimentar em população adulta do Distrito Federal.

\section{MÉTODOS}

Recomenda-se uma amostra entre 50 e 100 pessoas para cada grupo demográfico ${ }^{2,5}$. Sendo assim, iniciaram a pesquisa 80 participantes, distribuídos em 41 homens e 39 mulheres. $\mathrm{Na}$ segunda etapa desses foram resgatadas 75 pessoas (38 homens e 37 mulheres) e, ao final, na terceira fase, permaneceram 69 indivíduos (34 homens e 35 mulheres). A amostra final correspondeu a $86 \%$ da amostra inicial. Os principais motivos para a não permanência até o final da pesquisa foram a troca de emprego e a mudança de números telefônicos ou de endereço. Isso inviabilizou o contato para a continuidade das entrevistas.

Foram convidados a participar voluntariamente da pesquisa, mediante consentimento pessoal, clientes de dois restaurantes industriais da cidade de Brasília (Distrito Federal, Brasil) pertencentes a dois órgãos públicos que atendem a população adulta, considerada sadia e economicamente ativa.

A confidencialidade dos dados foi assegurada. O Comitê de Ética em Pesquisa em Seres Humanos da Faculdade de Ciências da Saúde da Universidade de Brasília (UnB) aprovou a pesquisa.
As entrevistas foram realizadas por quatro alunas do curso de graduação em Nutrição da UnB, participantes do Programa Institucional de Bolsas de Iniciação Científica (PIBIC/CNPq), especificamente treinadas para tal tarefa.

O intervalo de tempo total do estudo compreendeu os meses de dezembro de 2002 a maio de 2003.

O recordatório foi aplicado em três momentos diferentes: do primeiro para o segundo, o intervalo médio de tempo foi de 82 dias, e do segundo para o terceiro, 97 dias.

O questionário de freqüência de consumo alimentar (QFCA) (em anexo) foi preenchido em dois momentos, juntamente com o primeiro e o terceiro recordatórios, ou seja, ao início e ao final da coleta de dados, com um intervalo total de aproximadamente 181 dias.

O instrumento de pesquisa utilizado consistiu de três partes: identificação, recordatório $24 \mathrm{~h}$ (R24h) e QFCA semiquantitativo. Estes dois últimos constituem-se os métodos de inquérito dietético que foram comparados - objeto desta pesquisa.

Na parte de identificação foram levantados dados gerais sobre o indivíduo, tais como sexo, idade, escolaridade e formas de contato.

O recordatório $24 \mathrm{~h}$ era composto de quatro colunas: a) refeição - horário; b) alimento consumido; c) quantidade - medida caseira; d) quantidade - $\mathrm{g} / \mathrm{ml}$. Todas as colunas eram abertas para preenchimento.

O questionário de freqüência de consumo alimentar foi construído a partir do questionário validado por Sichieri \& Everhart ${ }^{12}$. Para adaptá-lo à população do Distrito Federal, fez-se um estudo piloto com 50 indivíduos pertencentes à população alvo. A partir deste estudo, alguns itens alimentares foram alterados a fim de que se adequassem à realidade da pesquisa.

Os alimentos foram ordenados em sete grupos alimentares: cereais e leguminosas, óleos e gorduras, sobremesas e petiscos, carnes e ovos, leites e derivados, hortaliças e frutas e bebidas. Este último incluía refrigerantes e sucos indus- 
trializados. O suco in natura integrou o grupo das frutas. No grupo das hortaliças e frutas foram obtidos os dois representantes mais freqüentemente consumidos para cada entrevistado. Os quatro alimentos mais mencionados em cada item foram: a) folha crua: alface, rúcula, repolho e couve; b) folha refogada ou cozida: couve, repolho, espinafre e mostarda; c) vegetal cru: tomate, beterraba, cenoura e pepino; d) vegetal cozido: cenoura, abóbora, abobrinha e brócolis; e) frutas: mamão, maçã, laranja e banana.

Cada grupo alimentar possuía entre 8 e 10 itens, exceto o grupo do leite e derivados, com 3. O instrumento constou, ao todo, de 52 itens.

As categorias de freqüência de consumo incluíam: a) uma vez por dia; b) duas ou mais vezes por dia; c) quatro a seis vezes por semana; d) duas a três vezes por semana; e) uma vez por semana; f) uma vez por mês; g) duas ou mais vezes por mês; $h$ ) raramente ou nunca.

A porção média em medidas caseiras de consumo foi obtida para todos os alimentos da lista.

Com o auxílio de uma tabela de medidas caseiras $^{13}$, fez-se o cálculo para consumo diário de cada item, tanto no R24h quanto no QFCA. No caso do QFCA, relacionou-se também a freqüência de consumo de cada alimento, transformando-o para a base-dia.

A partir do programa Virtual Nutri ${ }^{14}$ foram calculadas as ingestões absolutas dos macronutrientes (carboidratos, proteínas e lipídeos), alguns micronutrientes (vitaminas A e C, cálcio, ferro e zinco), colesterol, fibras dietéticas e valor energético total.

Inicialmente, a transformação logarítmica neperiana foi utilizada em todos os nutrientes, pois os mesmos não apresentavam distribuição gaussiana. Médias e desvios-padrão foram obtidos para o R24h e para o QFCA.

O coeficiente de correlação intraclasse foi calculado para o QFCA e o coeficiente de correlação de Pearson foi obtido para os dados brutos e deatenuados. A deatenuação para corrigir a variabilidade intra-indivíduo foi obtida usando-se a fórmula sugerida por Beaton et al. ${ }^{15}$. Valores foram ajustados para energia usando-se o método residual.

Demais variáveis correspondem à análise da amostra segundo idade e grau de escolaridade.

\section{RES U LT A D OS}

Quanto à caracterização da amostra, a média de idade foi de 35,4 anos (desvio-padrão $11,8)$. Houve predominância da faixa etária de 35 a 50 anos, perfazendo um total de $40,6 \%$ da amostra. A média de anos totais de estudo foi de 13,6 anos (desvio-padrão 2,3). A maioria dos entrevistados referiu ser graduado ou estar cursando nível superior (58,0\%). O menor número de anos totais de estudo encontrado foi 10 , que corresponde ao penúltimo ano do ensino médio.

As médias de ingestão absoluta estimada, provenientes do QFCA, mostraram-se para a maioria dos nutrientes maiores do que as do R24h (Tabela 1).

Os coeficientes de correlação intraclasse (Tabela 2) variaram entre 0,43 e 0,76. Energia, proteína, lipídeo e colesterol obtiveram valores iguais ou superiores a 0,7 , enquanto vitamina $A$, vitamina $\mathrm{C}$ e fibra apresentaram valores iguais ou inferiores a 0,5.

Na Tabela 3 são apresentados os resultados quanto à análise pelo coeficiente de correlação de Pearson, nas formas bruta e deatenuada, não ajustada e ajustada para energia. Os coeficientes deatenuados, ajustados para a energia, mostraram uma variação do menor para o maior de 0,32 a 0,66 , sendo, respectivamente, colesterol e vitamina C. Valores iguais ou superiores a 0,5 foram encontrados para fibra, zinco, cálcio, energia, proteína, ferro e vitamina $C$. 
Tabela 1. Média de ingestão absoluta estimada de nutrientes para dois QFCA e três R24h. Brasília, 2002/2003.

\begin{tabular}{|c|c|c|c|c|c|c|}
\hline \multirow{2}{*}{ Nutrientes } & \multicolumn{2}{|c|}{ QFCA } & \multicolumn{2}{|c|}{ R24h } & \multicolumn{2}{|c|}{ Diferença (QFCA-R24h) } \\
\hline & $M$ & DP & $M$ & DP & $M$ & DP \\
\hline Energia (kcal) & 2200,0 & 890,29 & 2020,0 & 870,16 & 180,0 & 622,48 \\
\hline Proteína (g) & 95,2 & 41,53 & 85,4 & 44,45 & 9,8 & 32,46 \\
\hline Carboidrato (g) & 267,3 & 103,67 & 264,0 & 108,55 & 3,3 & 76,83 \\
\hline Lipídeo (g) & 83,3 & 44,83 & 69,1 & 38,07 & 14,2 & 34,66 \\
\hline Ferro (mg) & 16,5 & 7,06 & 14,4 & 6,36 & 2,2 & 5,06 \\
\hline Cálcio (mg) & 737,7 & 389,93 & 623,0 & 360,42 & 114,8 & 360,80 \\
\hline Vitamina C (mg) & 205,4 & 137,41 & 185,2 & 193,59 & 20,2 & 153,35 \\
\hline Vitamina A (mcg RE) & 1477,1 & 845,31 & 910,9 & 592,28 & 566,2 & 872,70 \\
\hline Zinco (mg) & 9,2 & 5,62 & 8,0 & 5,21 & 1,2 & 4,08 \\
\hline Colesterol (mg) & 452,0 & 349,40 & 239,8 & 174,56 & 212,1 & 323,05 \\
\hline Fibra (g) & 14,5 & 6,88 & 14,4 & 6,36 & 2,7 & 5,88 \\
\hline
\end{tabular}

QFCA=Questionário de Freqüência de Consumo Alimentar Semiquantitativo; R24h=Recordatório 24 horas; M=média; DP=Desvio-padrão.

Tabela 2. Medida da reprodutibilidade (coeficiente de correlação intraclasse) do QFCA. Brasília, 2002/2003.

\begin{tabular}{lcc}
\hline Nutrientes & QFCA & $($ IC 95\%) \\
\hline Energia (kcal) & 0,73 & $(0,60-0,83)$ \\
Proteína (g) & 0,70 & $(0,56-0,81)$ \\
Carboidrato (g) & 0,63 & $(0,46-0,75)$ \\
Lipídeo (g) & 0,76 & $(0,64-0,84)$ \\
Ferro (mg) & 0,67 & $(0,52-0,78)$ \\
Cálcio (mg) & 0,62 & $(0,46-0,75)$ \\
Vitamina C (mg) & 0,43 & $(0,22-0,61)$ \\
Vitamina A (mcg RE) & 0,50 & $(0,31-0,66)$ \\
Zinco (mg) & 0,68 & $(0,53-0,79)$ \\
Colesterol (mg) & 0,74 & $(0,61-0,83)$ \\
Fibra (g) & 0,43 & $(0,21-0,60)$ \\
\hline
\end{tabular}

QFCA=Questionário de Freqüência de Consumo Alimentar Semiquantitativo; IC=Intervalo de confiança.

\section{DISCUSSÃO}

Destaca-se, inicialmente, o pequeno número de estudos sobre validação de métodos de inquérito dietético no País. Conforme observado por Slater et al. ${ }^{5}$, existem apenas quatro estudos realizados no Brasil nesse sentido. O mesmo foi constatado por Crispim et al.2. Porém, pode-se acrescentar a esse rol o estudo de Fornés et al. ${ }^{16}$.

De acordo com o Censo Demográfico ${ }^{17}$, a média de anos deestudo das pessoas responsáveis pelos domicílios no Brasil foi de 5,7 anos e no Distrito Federal, 8,1. Na análise desta amostra,

Tabela 3. Medida da validade (coeficiente de correlação de Pearson) entre QFCA e R24h. Brasília, 2002/2003.

\begin{tabular}{|c|c|c|c|c|c|c|c|c|}
\hline \multirow{2}{*}{ Nutrientes } & \multicolumn{4}{|c|}{ Não ajustado } & \multicolumn{4}{|c|}{ Ajustado para energia } \\
\hline & Bruto & (IC 95\%) & Deatenuado & (IC 95\%) & Bruto & (IC 95\%) & Deatenuado & (IC 95\%) \\
\hline Energia (kcal) & 0,79 & $(0,68-0,87)$ & 0,81 & $(0,71-0,88)$ & & - & & - \\
\hline Proteína (g) & 0,66 & $(0,50-0,78)$ & 0,71 & $(0,57-0,81)$ & 0,52 & $(0,32-0,67)$ & 0,55 & $(0,36-0,70)$ \\
\hline Carboidrato (g) & 0,78 & $(0,66-0,86)$ & 0,84 & $(0,75-0,90)$ & 0,51 & $(0,31-0,66)$ & 0,55 & $(0,36-0,70)$ \\
\hline Lipídeo (g) & 0,72 & $(0,58-0,81)$ & 0,76 & $(0,64-0,84)$ & 0,42 & $(0,20-0,61)$ & 0,44 & $(0,23-0,61)$ \\
\hline Ferro (mg) & 0,73 & $(0,60-0,82)$ & 0,79 & $(0,68-0,87)$ & 0,51 & $(0,31-0,66)$ & 0,58 & $(0,40-0,72)$ \\
\hline Cálcio (mg) & 0,55 & $(0,36-0,70)$ & 0,59 & $(0,41-0,73)$ & 0,48 & $(0,28-0,64)$ & 0,52 & $(0,32-0,67)$ \\
\hline Vitamina C (mg) & 0,60 & $(0,43-0,73)$ & 0,66 & $(0,50-0,78)$ & 0,60 & $(0,43-0,73)$ & 0,66 & $(0,50-0,78)$ \\
\hline Vitamina A (mcg RE) & 0,38 & $(0,16-0,57)$ & 0,43 & $(0,22-0,61)$ & 0,33 & $(0,10-0,53)$ & 0,37 & $(0,14-0,55)$ \\
\hline Zinco (mg) & 0,69 & $(0,54-0,80)$ & 0,77 & $(0,65-0,85)$ & 0,45 & $(0,24-0,62)$ & 0,50 & $(0,30-0,66)$ \\
\hline Colesterol (mg) & 0,49 & $(0,29-0,65)$ & 0,54 & $(0,35-0,69)$ & 0,29 & $(0,05-0,49)$ & 0,32 & $(0,09-0,52)$ \\
\hline Fibra (g) & 0,56 & $(0,38-0,71)$ & 0,61 & $(0,43-0,74)$ & 0,46 & $(0,25-0,63)$ & 0,50 & $(0,30-0,66)$ \\
\hline
\end{tabular}

QFCA=Questionário de Freqüência de Consumo Alimentar Semiquantitativo; R24h=Recordatório 24 horas; IC=Intervalo de confiança. 
de residentes no Distrito Federal, predominantemente adultos, verifica-se elevado grau de escolaridade, uma vez que a média encontrada foi de 13,6 anos. Segundo Kristal et al. ${ }^{4}$, existe uma forte relação entre o grau de escolaridade e a fidedignidade das informações coletadas por questionários de freqüência alimentar. A confiabilidade das informações e a validade do método aumentam consideravelmente, à medida que se aumentam os anos de estudo. Sendo assim, pode-se afirmar que a presente amostra apresenta uma potencial vantagem em fornecer dados mais confiáveis.

O padrão de ingestão absoluta encontrado (Tabela1) foi maior do que o observado por Willett et al. ${ }^{9}$, em que os valores obtidos com o QFCA foram maiores do que os observados nas médias dos registros alimentares. Kroke et al. ${ }^{8}$ também observaram uma tendência do QFCA em obter estimativas maiores para ingestão de energia, proteína total e carboidrato total. Contudo, no caso do colesterol, estes pesquisadores obtiveram uma estimativa menor para o QFCA, enquanto que na presente pesquisa foi verificado quase o dobro do valor encontrado nos recordatórios.

De acordo com Salvo \& Gimeno ${ }^{18}$, estudos de reprodutibilidade e validade de inquéritos dietéticos apresentam com freqüência valores de coeficientes de correlação entre 0,5 e 0,7 e estes são considerados como detentores de razoável reprodutibilidade e validade. Willett ${ }^{19}$ afirma ainda que os coeficientes de correlação devem encontrar-se na faixa de 0,4 a 0,7 para possuirem validação aceitável.

As informações acerca da reprodutibilidade do método (Tabela 2) indicam melhor consistência dos dados obtidos para energia, proteína e colesterol. Vitamina $\mathrm{C}$ e fibra obtiveram o pior resultado, indicando variação de ingestão intraindivíduo desses nutrientes. Corroborando estes achados, os intervalos de confiança (IC 95\%) encontrados (Tabela 2) revelaram grandes variações para as vitaminas $\mathrm{A}$ e $\mathrm{C}$ e fibras. Variações casuais, associadas à finalização dos questionários e a uma mudança real na ingestão da dieta regular, são considerados dois fatores que podem afetar a reprodutibilidade do QFCA. Flutuações sazonais nos hábitos alimentares podem ser outra fonte de variação ${ }^{20}$. Contudo, os valores dos coeficientes de correlação intraclasse obtidos, de maneira geral, apresentaram-se maiores do que os observados em outros estudos 3,9,18.

Quanto à validade (Tabela 3), observou-se que os coeficientes de correlação de Pearson, deatenuados e ajustados para energia, foram semelhantes aos de outras pesquisas com metodologia parecida ${ }^{8,21-23}$. Esse coeficiente é bastante utilizado em pesquisas de validação de métodos de inquérito dietético ${ }^{18}$.

Notou-se uma tendência de coeficientes menores para colesterol, em comparação a esses estudos, sendo esse também o nutriente com menor valor obtido (ajustado para energia e deatenuado). Vitamina $A$ também teve valor inferior a 0,4 . No entanto, a vitamina $C$ comportou-se diferentemente do esperado, obtendo o melhor valor.

Sobre o cálcio, Waib \& Burini23 notaram que o recordatório 24 horas pode ser um método satisfatório de obtenção da ingestão desse micronutriente, desde que se tenha uma amostra razoável. Entretanto, acredita-se que o recordatório tenha a tendência de nivelar as ingestões, de forma a superestimar as dietas baixas e a subestimar as altas. Os autores recomendam a associação com outros métodos, como, por exemplo, o questionário de freqüência alimentar, para enriquecer e validar os dados obtidos. Nesta pesquisa, o cálcio apresentou valor superior a 0,4, demonstrando validade aceitável ${ }^{19}$.

Uma explicação para o fato de colesterol e vitamina A terem obtido valores inferiores a 0,4 e IC 95\% com grande amplitude, encontra-se na variabilidade da ingestão dietética individual. 0 recordatório avalia a ingestão de apenas um único dia por vez, enquanto o QFCA observa um padrão de ingestão por um período maior de tempo, geralmente de três a seis meses ${ }^{24}$. Provavelmente, para captar com maior precisão a ingestão de alguns nutrientes seriam necessárias mais replicações de recordatórios do que de QFCA $A^{6,19}$. 
Quantos aos intervalos de confiança, tanto para o coeficiente de correlação intraclasse quanto para o coeficiente de correlação de Pearson, algumas considerações estatísticas podem ser feitas. O tamanho da amostra deste estudo está de acordo com o que é normalmente recomendado para pesquisas com esta metodologia2,5. Todavia, para todos os casos, os IC de $95 \%$ poderiam ser influenciados pelo tamanho da amostra e pelo próprio valor de coeficiente obtido. Quanto maior for o tamanho da amostra e quanto mais próximo de um for a medida de correlação, menor será a amplitude do intervalo de confiança. Como todos os cálculos foram baseados em uma amostra de tamanho fixo igual a 69, os intervalos de confiança passam a ser influenciados pelo valor da medida - maiores amplitudes de intervalo dar-se-ão para valores mais afastados de um. Caso a amostra fosse aumentada, a precisão das estimativas das medidas seria menor e obedeceria ao princípio de quanto mais próximos de 1 fossem os valores de coeficientes encontrados, menores seriam os intervalos de confiança. Contudo, há de considerar também que nenhum intervalo incluiu o zero, logo, todos obtiveram correlação positiva e significante.

Além do fato de três recordatórios, possivelmente, serem insuficientes para mensurar a ingestão habitual da maior parte dos micronutrientes ${ }^{6,19}$, e do tamanho da amostra influenciar a obtenção de coeficientes maiores para alguns nutrientes, acrescentam-se, como limitações do estudo, características inerentes aos métodos utilizados, como a dificuldade em conseguir tamanhos exatos para as porções consumidas, a dependência da memória dos entrevistados ${ }^{25} \mathrm{e} \mathrm{a}$ possibilidade de variações do hábito alimentar durante o tempo de estudo ${ }^{20}$.

Assim, para condução de estudos de consumo alimentar baseados em inquéritos e, a depender dos nutrientes a serem avaliados, deve-se considerar aumentar o tamanho da amostra e o número de replicações do R24h, de modo a minimizar possíveis distorções.

\section{CONCLUSÃO}

A obtenção de coeficientes de correlação expressivos para alguns nutrientes indica que 0 questionário de freqüência de consumo alimentar avaliado neste estudo constitui-se em um bom instrumento de pesquisa para estudos epidemiológicos em população adulta, podendo fornecer informações importantes para a implementação de ações na área de saúde e nutrição.

\section{A GRADECIMENTOS}

A todos os participantes da pesquisa e às bolsistas do Programa Institucional de Bolsas de Iniciação Científica (PIBIC), Ivana Aragão Lira Vasconcelos, Márcia Hiroko Ueda e Mariana Helcias Côrtes, pela colaboração na coleta e digitação dos dados.

\section{REFER Ê N CIAS}

1. Cintra IP, von Der Heyde MED, Schmitz BAS, Franceschini SCC, Taddei JAAC, Sigulem DM. Métodos de inquéritos dietéticos. Cad Nutr. 1997; 13:11-23

2. Crispim SP, Silva MMS, Ribeiro RCL. Validação de questionários de freqüência alimentar. Nutr Brasil. 2003; 2(5):286-90.

3. Boeing $H$, Bohlscheid-Thomas $S$, Voss $S$, Schneeweiss S, Wahrendorf J. The relative validity of vitamin intakes derived from a food frequency questionnaire compared to 24-hour recalls and biological measurements: results from the EPIC pilot study in Germany. Int J Epidemiol. 1997; 26(Suppl 1):S82-S90.

4. Kristal AR, Feng Z, Coates RJ, Oberman A, George $V$. Associations of race/ethnicity, education, and dietary intervention with the validity and reliability of a food frequency questionnaire. Am J Epidemiol. 1997; 146(10):856-69.

5. Slater B, Philippi ST, Marchioni DML, Fisberg RM. Validação de questionários de freqüência alimentar - QFA: considerações metodológicas. Rev Bras Epidemiol. 2003; 6(3):200-8.

6. Eck LH, Klesges LM, Klesges RC. Precision and estimated accuracy of two short-term food frequency questionnaires compared with recalls 
and records. J Clin Epidemiol. 1996; 49(10): 1195-200.

7. Hu FB, Rimm E, Smith-Warner SS, Feskanich D, Stampfer MJ, Ascheiro A, et al. Reproducibility and validity of dietary patterns assessed with a food-frequency questionnaire. Am J Clin Nutr. 1999; 69(2):243-9.

8. Kroke A, Klipstein-Grobusch K, Voss S, Möseneder J, Thielecke F, Noack R, et al. Validation of self-administered food-frequency questionnaire administered in the European Prospective Investigation into Cancer and Nutrition (EPIC) Study: comparison of energy, protein, and macronutrient intakes estimated with the doubly labeled water, urinary nitrogen, and repeated 24-h dietary recall methods. Am J Clin Nutr. 1999; 70(4):439-47.

9. Willett WC, Sampson L, Meir JS, Rosner B, Bain C, Witschi J, et al. Reprodutibility and validity of a semiquantitative food frequency questionnaire. Am J Epidemiol. 1985; 122(1):51-65.

10. Caan BJ, Slattery ML, Potter J, Quesenberry Jr. CP, Coates AO, Schaffer DM. Comparison of the Block and the Willett self-administered semiquantitative food frequency questionnaires with an interviewer-administered dietary history. Am J Epidemiol. 1998; 148(12):1137-47.

11. Wirfält AKE, Jeffery RW, Elmer PJ. Comparison of food frequency questionnaires: the reduced block and willet questionnaires differ in ranking on nutrient intakes. Am J Epidemiol. 1998; 148(12): 1148-56.

12. Sichieri R, Everhart JE. Validity of a Brazilian food frequency questionnaire against dietary recalls and estimated energy intake. Nutr Res. 1998; 18(10):1649-59.

13. Pinheiro ABV, Lacerda EMA, Benzecry EH, Gomes MC, Costa VM. Tabela para avaliação de consumo em medidas caseiras. 4. ed. São Paulo: Atheneu; 2001.

14. Philippi ST, Szarfarc SC, Latterza AR. Virtual Nutri [computer program], versão1.0, for Windows. São Paulo; Departamento de Nutrição, Faculdade de Saúde Pública, USP; 1996.

15. Beaton GH, Milner J, Corey P, McGuire V, Cousins $M$, Steawart $E$, Ramos $M$, et al. Sources of variance in 24-hour dietary recall data: implications for nutrition study design and interpretation. Am J Clin Nutr 1979; 32(12): 2546-9.

16. Fornés NS, Stringhini MLF, Elias BM. Reproducibility and validity of a food-frequency questionnaire for use among low-income Brazilian workers. Publ Health Nutr. 2003; 6(8):821-7.

17. Instituto Brasileiro de Geografia e Estatística. Censo Demográfico 2000. Indicadores Sociais Municipais: 2000. [acesso em 11 nov. 2003]. Disponível em: http://www.ibge.gov.br

18. Salvo VLMA, Gimeno SGA. Reprodutibilidade e validade do questionário de freqüência de consumo de alimento. Rev Saúde Pública. 2002; 36(4):505-12.

19. Willet WC, Buzzard IM. Foods and nutrients. In: Willet WC. Nutritional epidemiology. New York: Oxford University Press; 1998.

20. Tsubono Y, Nishino Y, Fukao A, Hisamichi S, Tsugane $\mathrm{S}$. Temporal change in the reproducibility of a self-administered food frequency questionnaire. Am J Epidemiol. 1995; 142(11): 1231-5.

21. Bohlscheid-Thomas S, Hoting I, Boeing H, Wahrendorf J. Reproducibility and relative validity of energy and macronutrient intake of a food frequency questionnaire developed for the German Part of the EPIC Project. Int J Epidemiol. 1997; 26(Suppl 1): S71-S81

22. Ocké MC, Bueno-de-Mesquita HB, Pols MA, Smit HA, Van Staveren WA, Kromhout D. The Dutch EPIC food frequency questionnaire. II. relative validity and reproducibility for nutrients. Int J Epidemiol. 1997; 26(Suppl 1):S49-58.

23. Waib PH, Burini RC. Aplicação dos métodos de inquérito alimentar na avaliação da ingestão de cálcio em estudos epidemiológicos. Rev Nutr. 1990; 3(2):143-57.

24. Crispim SP, Franceschini SCC, Priore SE, Fisberg RM. Validação de inquéritos dietéticos: uma revisão. Nutrire. 2003; 26:127-41.

25. Fisberg RM, Slater B, Marchioni DML, Martini LA. Inquéritos alimentares: métodos e bases científicos. Barueri: Manole; 2005.

Recebido em: 7/1/2005

Versão final reapresentada em: 27/4/2006 Aprovado em: 3/8/2006 
ANEXO

QUESTIONÁRIO DE FREQÜÊNCIA DE CONSUMO ALIMENTAR (QFA)

\begin{tabular}{|c|c|c|c|c|c|c|c|c|c|}
\hline \multirow[b]{2}{*}{ Produtos } & \multirow[b]{2}{*}{$\begin{array}{c}\text { Porção } \\
\text { consumida } \\
(n \% \text { descrição) }\end{array}$} & \multicolumn{8}{|c|}{ Freqüência } \\
\hline & & $\begin{array}{l}1 \text { vez } \\
\text { por } \\
\text { dia }\end{array}$ & $\begin{array}{c}2 \text { ou mais } \\
\text { vezes por } \\
\text { dia }\end{array}$ & $\begin{array}{c}5 \text { a } 6 \\
\text { vezes por } \\
\text { semana }\end{array}$ & \begin{tabular}{|c|}
2 a 4 \\
vezes por \\
semana \\
\end{tabular} & \begin{tabular}{|c}
1 vez \\
por \\
semana
\end{tabular} & \begin{tabular}{|c|}
1 a 3 \\
vezes \\
por mês
\end{tabular} & $\mathrm{R} / \mathrm{N}$ & Qtd. $\mathrm{g} / \mathrm{ml}$ \\
\hline
\end{tabular}

LEITE E DERIVADOS

\begin{tabular}{|l|l|l|l|l|l|l|l|l|l|}
\hline $\begin{array}{l}\text { Leite desnatado ou } \\
\text { semi-desnatado }\end{array}$ & & & & & & & & & \\
\hline Leite integral & & & & & & & & & \\
\hline logurte & & & & & & & & & \\
\hline Queijo branco (minas/frescal & & & & & & & & & \\
\hline $\begin{array}{l}\text { Queijo amarelo (prato/ } \\
\text { mussarela) }\end{array}$ & & & & & & & & & \\
\hline Requeijão & & & & & & & & & \\
\hline
\end{tabular}

\section{CARNES E OVOS}

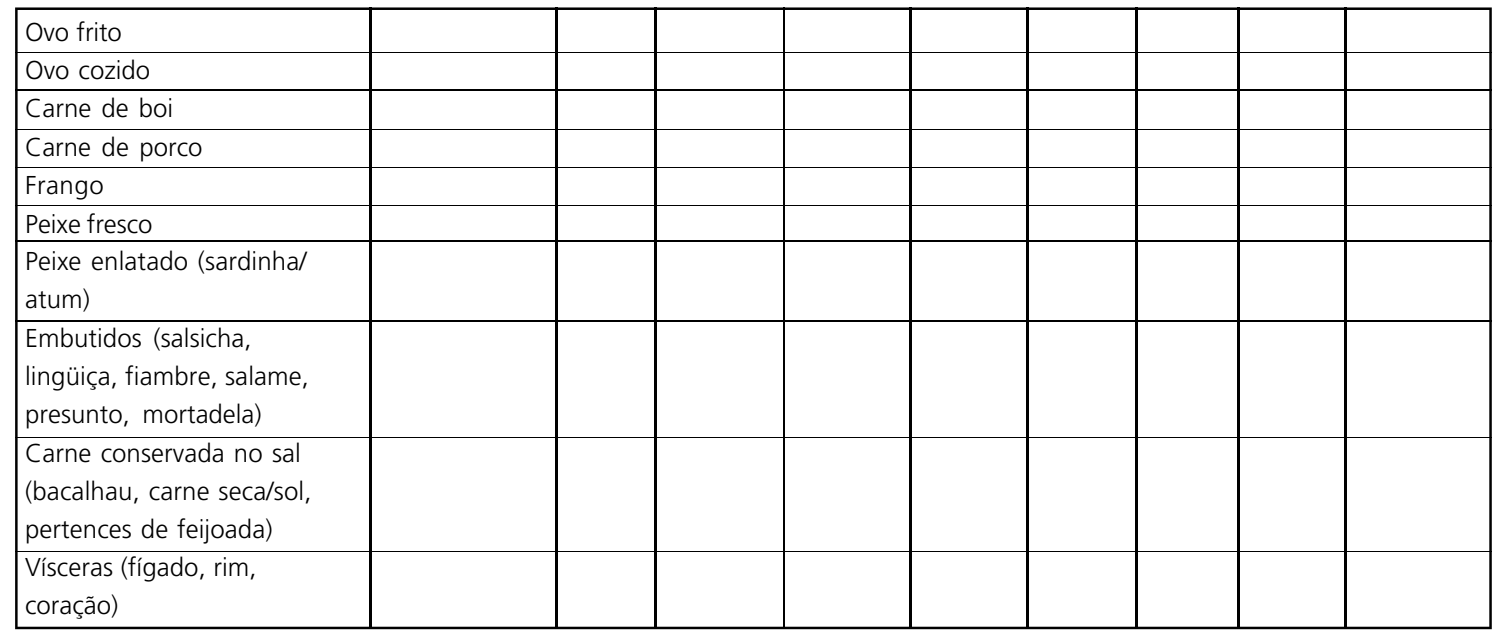

ÓLEOS

\begin{tabular}{|l|l|l|l|l|l|l|l|l|l|}
\hline Azeite & & & & & & & & & \\
\hline Molho para salada & & & & & & & & & \\
\hline Bacon e toucinho & & & & & & & & & \\
\hline Manteiga & & & & & & & & & \\
\hline Margarina & & & & & & & & & \\
\hline Maionese & & & & & & & & & \\
\hline
\end{tabular}

\section{PESTISCOS E ENLATADOS}

\begin{tabular}{|l|l|l|l|l|l|l|l|l|l|}
\hline Snacks (batata-frita, & & & & & & & & & \\
\hline $\begin{array}{l}\text { sanduíches, pizza, esfiha, } \\
\text { salgadinhos, cheetos, } \\
\text { amendoim) }\end{array}$ & & & & & & & & & \\
\hline $\begin{array}{l}\text { Enlatados (milho, ervilha, } \\
\text { palmito, azeitona) }\end{array}$ & & & & & & & & & \\
\hline
\end{tabular}

\section{CEREAIS/ LEGUMINOSAS}

\begin{tabular}{|l|l|l|l|l|l|l|l|l|l|}
\hline Arroz integral & & & & & & & & & \\
\hline Arroz polido & & & & & & & & & \\
\hline Pão integral & & & & & & & & & \\
\hline Pão francês/forma & & & & & & & & & \\
\hline Biscoito salgado & & & & & & & & & \\
\hline Biscoito doce & & & & & & & & & \\
\hline Bolos & & & & & & & & & \\
\hline
\end{tabular}


QUESTIONÁRIO DE FREQÜÊNCIA DE CONSUMO ALIMENTAR (QFA)

\begin{tabular}{|c|c|c|c|c|c|c|c|c|c|}
\hline \multirow[b]{2}{*}{ Produtos } & \multirow[b]{2}{*}{$\begin{array}{c}\text { Porção } \\
\text { consumida } \\
\text { (n\% descrição) }\end{array}$} & \multicolumn{8}{|c|}{ Freqüência } \\
\hline & & $\begin{array}{l}1 \text { vez } \\
\text { por } \\
\text { dia }\end{array}$ & \begin{tabular}{|c|}
$\begin{array}{c}2 \text { ou mais } \\
\text { vezes por } \\
\text { dia }\end{array}$ \\
\end{tabular} & \begin{tabular}{|c|}
5 a 6 \\
vezes por \\
semana \\
\end{tabular} & \begin{tabular}{|c|}
2 a 4 \\
vezes por \\
semana \\
\end{tabular} & $\begin{array}{c}1 \mathrm{vez} \\
\text { por } \\
\text { semana }\end{array}$ & \begin{tabular}{|c|}
1 a 3 \\
vezes \\
por mês
\end{tabular} & $\mathrm{R} / \mathrm{N}$ & Qtd. $\mathrm{g} / \mathrm{ml}$ \\
\hline \multicolumn{10}{|c|}{ CEREAIS/ LEGUMINOSAS } \\
\hline \multicolumn{10}{|l|}{ Macarrão } \\
\hline Feijão & & & & & & & & & \\
\hline
\end{tabular}

HORTALIÇAS E FRUTAS

\begin{tabular}{|l|l|l|l|l|l|l|l|l|l|}
\hline Folha crua: & & & & & & & & & \\
\hline- & & & & & & & & & \\
\hline Folha refogada/ cozida: & & & & & & & & \\
\hline- & & & & & & & & & \\
\hline Hortaliça crua: & & & & & & & & & \\
\hline- & & & & & & & & & \\
\hline Hortaliça cozida: & & & & & & & & & \\
\hline-
\end{tabular}

SOBREMESAS E DOCES

\begin{tabular}{|l|l|l|l|l|l|l|l|l|l|}
\hline Sorvete & & & & & & & & & \\
\hline Tortas & & & & & & & & & \\
\hline Geléia & & & & & & & & & \\
\hline Doces/balas & & & & & & & & & \\
\hline $\begin{array}{l}\text { Chocolates/achocolatados/ } \\
\text { bombom }\end{array}$ & & & & & & & & & \\
\hline
\end{tabular}

BEBIDAS

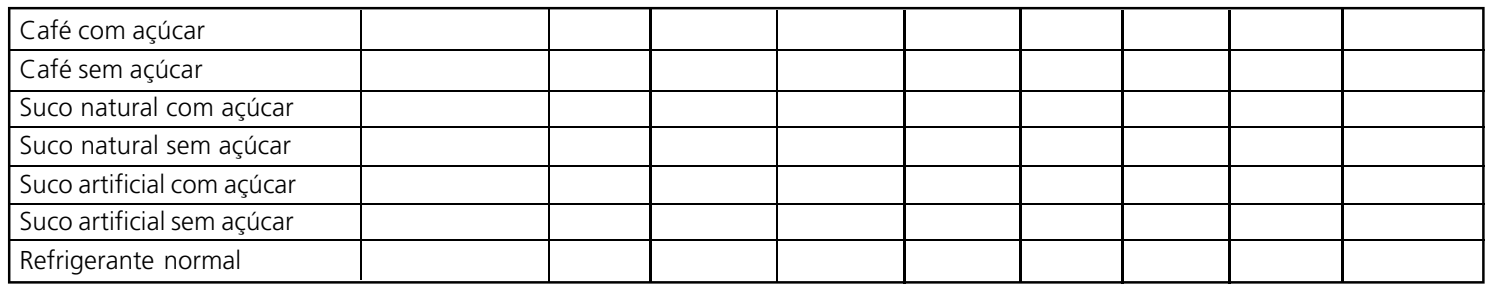

PRODUTOS DIET E LIGHT

\begin{tabular}{|l|l|l|l|l|l|l|l|l|l|}
\hline Adoçante & & & & & & & & & \\
\hline Margarina & & & & & & & & & \\
\hline Requeijão/iogurte & & & & & & & & & \\
\hline Refrigerante & & & & & & & & & \\
\hline
\end{tabular}

